\title{
An efficient Newton-type method with fifth-order convergence for solving nonlinear equations
}

\author{
LIANG FANG ${ }^{1,2}$, LI SUN ${ }^{2}$ and GUOPING $\mathrm{HE}^{2,3}$ \\ ${ }^{1}$ Department of Mathematics and System Science, Taishan University, 271021, Tai'an, China \\ ${ }^{2}$ Department of Mathematics, Shanghai Jiaotong University, 200240, Shanghai, China \\ ${ }^{3}$ College of Information Science and Engineering, \\ Shandong University of Science and Technology, 266510, Qingdao, China
}

E-mail: fangliang3@163.com

\begin{abstract}
In this paper, we present an efficient Newton-like method with fifth-order convergence for nonlinear equations. The algorithm is free from second derivative and it requires four evaluations of the given function and its first derivative at each iteration. As a consequence, its efficiency index is equal to $\sqrt[4]{5}$ which is better than that of Newton's method $\sqrt{2}$. Several examples demonstrate that the presented algorithm is more efficient and performs better than Newton's method.
\end{abstract}

Mathematical subject classification: 41A25, 65H05, 65D99.

Key words: Newton's method, iterative method, nonlinear equations, order of convergence.

\section{Introduction}

We consider iterative methods to find a simple root $\alpha$ of a nonlinear equation $f(x)=0$, where $f: D \subseteq \mathcal{R} \rightarrow \mathcal{R}$ is a scalar function and it is sufficiently smooth in a neighborhood of $\alpha$, and $D$ is an open interval.

It is well known that classical Newton's method (named NM for simplicity)

$$
x_{n+1}=x_{n}-\frac{f\left(x_{n}\right)}{f^{\prime}\left(x_{n}\right)}
$$

\#747/07. Received: 12/XII/07. Accepted: 06/VI/08. 
is one of basic and important iterative methods for solving nonlinear equations and it converges quadratically [1] in a neighborhood of $\alpha$.

Recently, some fifth-order iterative methods have been proposed and analyzed for solving nonlinear equations. These methods improve some classical methods such as Newton's method, Cauchy's method and Halley's method [2-6]. It is said that the improved methods are efficient and can compete with Newton's method. For more details about them see [2-6] and the references therein. It should be noted that most of these fifth-order methods depend on the second derivative which strictly reduces their practical applications.

Motivated by the recent activities in this direction, we present and analyze a new fifth-order iterative method in this paper.

The paper is organized as follows. In section 2, we propose the new method and analyze its convergence. Some numerical results are given in section 3 . Finally, we draw some conclusions in section 4.

\section{The new method and its convergence analysis}

Consider the iteration scheme (FLM)

$$
x_{n+1}=y_{n}-\frac{5 f^{\prime 2}\left(x_{n}\right)+3 f^{\prime 2}\left(y_{n}\right)}{f^{\prime 2}\left(x_{n}\right)+7 f^{\prime 2}\left(y_{n}\right)} \cdot \frac{f\left(y_{n}\right)}{f^{\prime}\left(x_{n}\right)},
$$

where $y_{n}=x_{n}-\frac{f\left(x_{n}\right)}{f^{\prime}\left(x_{n}\right)}$.

For the method defined by (2), we have the following result.

Theorem 2.1. Assume that the function $f: D \subseteq \mathcal{R} \rightarrow \mathcal{R}$ has a single root $\alpha \in$ $D$, where $D$ is an open interval. Assume furthermore that $f(x)$ is a sufficiently differentiable function in the neighborhood of $\alpha$, then the order of convergence of the method FLM defined by (2) is five, and it satisfies the error equation

$$
e_{n+1}=\left(-c_{2}^{2} c_{3}+\frac{7}{2} c_{2}^{4}\right) e_{n}^{5}+O\left(e_{n}^{6}\right)
$$

Proof. Let $\alpha$ be the simple root of $f(x), c_{k}=f^{(k)}(\alpha) /\left(k ! f^{\prime}(\alpha)\right), k=2,3, \ldots$ and $e_{n}=x_{n}-\alpha$. Consider the iteration function $F(x)$ defined by

$$
F(x)=y(x)-\frac{5 f^{\prime 2}(x)+3 f^{\prime 2}(y(x))}{f^{\prime 2}(x)+7 f^{\prime 2}(y(x))} \frac{f(y(x))}{f^{\prime}(x)},
$$


where

$$
y(x)=x-\frac{f(x)}{f^{\prime}(x)} .
$$

By some computations using Maple we can obtain

$$
\begin{gathered}
F(\alpha)=\alpha, F^{(i)}(\alpha)=0, i=1, \ldots, 4, \\
F^{(5)}(\alpha)=\frac{-20 f^{\prime}(\alpha) f^{\prime \prime 2}(\alpha) f^{(3)}(\alpha)+105 f^{\prime \prime 4}(\alpha)}{4 f^{\prime 4}(\alpha)}=-120 c_{2}^{2} c_{3}+420 c_{2}^{4} .
\end{gathered}
$$

On the other hand, from the Taylor expansion of $F\left(x_{n}\right)$ around $\alpha$, we have

$$
\begin{aligned}
x_{n+1}= & F\left(x_{n}\right) \\
= & F(\alpha)+\sum_{i=1}^{4} \frac{F^{(i)}(\alpha)}{i !} F^{(i)}(\alpha)\left(x_{n}-\alpha\right)^{i} \\
& +\frac{F^{(5)}(\alpha)}{5 !}\left(x_{n}-\alpha\right)^{5}+0\left(\left(x_{n}-\alpha\right)^{6}\right) \\
= & \alpha+\left(-c_{2}^{2} c_{3}+\frac{7}{2} c_{2}^{4}\right) e_{n}^{5}+O\left(e_{n}^{6}\right) .
\end{aligned}
$$

Therefore, we obtain $e_{n+1}=\left(-c_{2}^{2} c_{3}+3.5 c_{2}^{4}\right) e_{n}^{5}+O\left(e_{n}^{6}\right)$, which means that the method defined by (2) is fifth-order convergent.

Now, we consider efficiency index defined as $p^{\frac{1}{\omega}}$, where $p$ is the order of the method and $\omega$ is the number of function evaluations required by the method at each iteration. It's easy to see that our method FLM has the efficiency index $\sqrt[4]{5}$ which is better than that of Newton's method $\sqrt{2}$.

\section{Numerical results}

In this section, we employ the new method FLM to solve some nonlinear equations and compare it with NM, (PPM) [7] defined by

$$
x_{n+1}=x_{n}-\frac{f\left(x_{n}\right)+f\left(y_{n}\right)}{f^{\prime}\left(x_{n}\right)},
$$

and two-step method (TSM)

$$
x_{n+1}=y_{n}-\frac{f\left(y_{n}\right)}{f^{\prime}\left(y_{n}\right)},
$$


where $y_{n}=x_{n}-\frac{f\left(x_{n}\right)}{f^{\prime}\left(x_{n}\right)}$.

All experiments were performed on a personal computer (IBM R40e) with Intel(R) Pentium(R) 4 CPU $2.00 \mathrm{GHz}$ and $512 \mathrm{MB}$ memory. The operating system was Windows XP (SP2) and the implementations were done in MATLAB 7.0.1 with double precision. We use the following stopping criteria for computer programs: $(i)\left|f\left(x_{n}\right)\right|<1 . E-14$, and $(i i)\left|x_{n+1}-x_{n}\right|<1 . E-14$.

Displayed in Table 1 are the order of convergence of different methods. The number of iterations (IT) and function evaluations (NFE) required such that the stopping criterion is satisfied are listed in Table 2. Table 3 gives the comparison of number of operations needed for obtaining solutions.

\begin{tabular}{|c|c|c|c|c|}
\hline & NM & PPM & TSM & FLM \\
\hline Order & 2 & 3 & 4 & 5 \\
\hline
\end{tabular}

Table 1 - Order of convergence of different iterative methods.

\begin{tabular}{|c|c|c|c|c|c|c|c|c|c|}
\hline & & \multicolumn{5}{|c|}{ IT } & \multicolumn{4}{|c|}{ NFE } \\
\hline$f(x)$ & $x_{0}$ & NM & PPM & TSM & FLM & NM & PPM & TSM & FLM \\
\hline$f_{1}(x)$ & -0.3 & 53 & 49 & 27 & 21 & 106 & 147 & 108 & 84 \\
\hline$f_{1}(x)$ & 1.75 & 5 & 3 & 3 & 2 & 10 & 9 & 12 & 8 \\
\hline$f_{2}(x)$ & -1.9 & 10 & 7 & 5 & 4 & 20 & 21 & 20 & 16 \\
\hline$f_{2}(x)$ & 5.5 & 9 & 6 & 5 & 4 & 18 & 18 & 20 & 16 \\
\hline$f_{3}(x)$ & -2.1 & 9 & 6 & 5 & 4 & 18 & 18 & 20 & 16 \\
\hline$f_{3}(x)$ & -2.5 & 11 & 8 & 6 & 5 & 22 & 24 & 24 & 20 \\
\hline
\end{tabular}

Table 2 - Comparison of IT and NFE of different iterative methods.

We used the following test functions and display the approximate zeros $x^{*}$ found up to the $14^{\text {th }}$ decimal place.

$$
\begin{aligned}
& f_{1}(x)=x^{3}+4 x^{2}-10, \quad x^{*}=1.36523001341410 . \\
& f_{2}(x)=x^{3}-2 x^{2}+x-1, \quad x^{*}=1.75487766624670 . \\
& f_{3}(x)=x e^{x^{2}}-\sin ^{2}(x)+3 \cos (x)+5, \quad x^{*}=-1.20764782713092 .
\end{aligned}
$$




\begin{tabular}{|c|c|c|c|c|c|c|c|c|c|}
\hline & & \multicolumn{5}{|c|}{+ or -} & \multicolumn{5}{c|}{$\times$ or $\div$} \\
\hline$f(x)$ & $x_{0}$ & NM & PPM & TSM & FLM & NM & PPM & TSM & FLM \\
\hline$f_{1}(x)$ & -0.3 & 212 & 539 & 324 & 294 & 212 & 343 & 216 & 252 \\
\hline$f_{1}(x)$ & 1.75 & 20 & 33 & 36 & 28 & 20 & 21 & 24 & 24 \\
\hline$f_{2}(x)$ & -1.9 & 60 & 77 & 60 & 56 & 40 & 49 & 40 & 48 \\
\hline$f_{2}(x)$ & 5.5 & 54 & 66 & 60 & 56 & 36 & 42 & 40 & 48 \\
\hline
\end{tabular}

Table 3 - Comparison of number of operations of different iterative methods.

From Table 1 and 3, we can see that, the algorithm FLM increase three order than classical Newton's method, but, only at the cost of small number of operations. We know, generally speaking, the increasing of order of convergence is more or less built on the cost of number of operations.

The computational results show that the present method requires less IT and NFE than NM, PPM and TSM as far as the numerical results are concerned. Therefore, the new method is of practical interest.

\section{Conclusions}

We present a new modification of Newton-type method with fifth-order convergence for solving nonlinear equations. At each iteration, it requires four evaluations of the function and its first derivative. Analysis of efficiency shows that the new algorithm is more efficient and it performs better than classical Newton's method and some other methods.

Acknowledgements. The authors would like to thank the anonymous referees for there constructive suggestions and criticisms that led to an improved presentation.

\section{REFERENCES}

[1] A.M. Ostrowski, Solution of Equations in Euclidean and Banach Space. Academic Press, New York (1973)

[2] M.A. Noor and K.I. Noor, Fifth-order iterative methods for solving nonlinear equations. Appl. Math. Comput., 188 (2007), 406-410. 
[3] J. Kou and Y. Li, The improvements of Chebyshev-Halley methods with fifth-order convergence. Appl. Math. Comput., 188 (2007), 143-147.

[4] M. Grau and M. Noguera, A Variant of Cauchy's Method with Accelerated Fifth-Order Convergence. Applied Mathematics Letters, 17 (2004), 509-517.

[5] J. Kou, Y. Li and X. Wang, Some modifications of Newton's method with fifth-order convergence. Appl. Math. Comput., 209 (2007), 146-152.

[6] J. Kou, The improvements of modified Newton's method. Appl. Math. Comput., 189 (2007), 602-609.

[7] F.A. Potra and V. Pták, Nondiscrete induction and iterative processes. Research Notes in Mathematics, vol. 103, Pitman, Boston (1984). 